\title{
A cost-utility analysis of small bite sutures versus large bite sutures in the closure of midline laparotomies in the United Kingdom National Health Service
}

This article was published in the following Dove Press journal: ClinicoEconomics and Outcomes Research

\section{Shyam Ajay Gokani' \\ Karl O Elmqvist ${ }^{\prime}$ \\ Osman El-Koubani' \\ Javier Ash' \\ Sudeep K Biswas' \\ Maxime Rigaudy ${ }^{2}$}

'Faculty of Medicine, Imperial College London, London, UK; ${ }^{2}$ Hull York

Medical School, University of York, York, UK
Correspondence: Shyam Ajay Gokani Faculty of Medicine, Imperial College London, South Kensington Campus London, SW7 2AZ, UK

Email shyamg@live.co.uk
Purpose: This study aimed to perform an economic evaluation of small bite sutures versus large bite sutures in the closure of midline laparotomies in the United Kingdom National Health Service (NHS).

Methods: A cost-utility analysis was conducted using data from a systematic literature review. Large bite sutures placed $10 \mathrm{~mm}$ from the wound edge were compared to small bite sutures 3-6 $\mathrm{mm}$ from the wound edge. The analysis used a 3-year time horizon in order to take into account complications including incisional hernias and surgical site infections (SSIs). Cost and benefit data were considered from the perspective of the NHS. A two-way sensitivity analysis was conducted to assess the impact of a variation in the clinical effectiveness of small bite sutures. Results: The incremental cost-effectiveness ratio was calculated to be $-£ 482.61$ per qualityadjusted life year (QALY) using the proposed small bite suture technique, indicating a cost saving to the NHS. Sensitivity analysis demonstrated that small bites are a cost-neutral technique provided that the cost of using small bites is less than $£ 98$ per patient. Small bites cost less than $£ 20,000$ /QALY when they reduce either the rate of SSIs by more than $15 \%$ or the rate of hernias by more than $3.4 \%$.

Conclusion: This study proposes that small bite sutures should become the mainstay suturing technique in the closure of midline laparotomies, replacing large bite sutures, which dominate current practice. The financial savings accompanied by the decrease in SSI rates and herniation warrant the use of this new technique. The sensitivity analysis demonstrates that findings hold true for a wide range of levels of clinical effectiveness for small bites.

Keywords: suture technique, economic evaluation, surgical site infection, incisional hernia

\section{Introduction}

Midline laparotomies are a common type of surgical operation aimed at gaining quick and safe access to the abdominal cavity. They use a midline vertical incision, typically from the xiphoid process to the umbilicus, that can be extended further if required. ${ }^{1,2}$ The simplicity and ease of the incision explain its use in multiple types of surgery, but it is most typical in exploratory and emergency operations. Approximately $30-50,000$ emergency midline laparotomies were performed in the UK in 2013. ${ }^{3}$ Despite being the most common way to gain access to the abdominal cavity, patients undergoing this surgical procedure are still at significant risk of postoperative complications. ${ }^{4}$ These most commonly include surgical site infections (SSIs), incisional hernias, wound dehiscence and bowel obstruction, among many others. ${ }^{5,6}$ 
The wound closure technique has been investigated as a way surgeons can reduce SSIs and incisional hernias, the most common postoperative complications, independently of other known risk factors such as age and weight. ${ }^{4,7}$ Many varieties of suture technique have been investigated such as single or layered stitching, differences in suture material, knotting techniques and the tension on the stitch. However, most recent studies have concentrated on the effect that suture length to wound length (SL:WL) ratio and bite size can have on SSI and incisional hernia rates. ${ }^{5,8,9}$

The focus of this study is limited to bite size. It is well documented that a SL:WL ratio of $\geq 4$ reduces the rate of incisional hernias. ${ }^{10}$ The effect of this ratio had not previously been known, and it has been argued that non-adherence to this ratio for obese patients is the reason that a higher proportion develops incisional hernias. ${ }^{11}$ Large bite sutures inserted $\sim 10 \mathrm{~mm}$ from the incision are the current standard in closure of midline laparotomies. ${ }^{4,12}$ This is due to evidence from previous studies demonstrating that smaller bites produce a weaker closure. ${ }^{13}$ However, recent evidence, published in The Lancet, demonstrates that this may be incorrect due to the researchers not taking the SL:WL ratio into account when analyzing the effect of small bites. ${ }^{5,14}$ When the SL:WL ratio is kept constant, small bites of 3-6 mm from the wound edge (instead of $10 \mathrm{~mm}$ ) have been shown to halve the rate of SSI and almost quarter the rate of incisional hernias that occur postoperatively. ${ }^{5,7,9}$

Following on from large recent studies into the effect of bite size, a cost-utility study for the UK is required to observe whether this change in practice is feasible and acceptable in the health service.

\section{Study objectives}

There is an increasing demand on the United Kingdom National Health Service (NHS), namely with respect to workforce and funds, coupled with the staggeringly high demand from society for health care. It is important to identify areas whereby quality of care can be improved without compromising the cost-effectiveness of the interventions offered. This study therefore aims to complete the following objectives:

- To assess the cost implications of adopting small bite sutures as standard practice

- To assess the impact of adopting small bite sutures as standard practice on quality of life measures

Thus, by comparing both the cost and utility of small bite sutures, this report aims to perform a thorough economic evaluation of the adoption of small bite sutures to standard NHS practice.

\section{Methods \\ Choice of analysis}

A cost-utility analysis (CUA) was chosen to measure outcomes, as this would compare both the costs of the proposed technique and also the change in quality-adjusted life years (QALYs). QALYs are obtained by quantifying changes in both quality of life as well as length of life, using standardized questionnaires, and then multiplying the two. CUA is the analytical method used by the National Institute of Health and Clinical Excellence (NICE) in the UK, and internationally, and so allows for comparability with other countries. ${ }^{15}$

Altering bite size can improve quality of life by reducing the rates of long-term complications, such as incisional hernias, and thus affect patient outcomes. By measuring the difference in costs and dividing by the difference in effects (derived from QALYs) between the two interventions, the incremental cost-effectiveness ratio (ICER) is found, which is compared against a threshold in order to see whether the small bites are more cost-effective than large bites.

The risk of some postoperative complications including dehiscence, hospital-acquired infections, hemorrhage, UTIs and bowel obstruction, and other extremes such as death were found to be equivalent for both types of suture technique. ${ }^{5}$ However, rates of SSI and incisional hernia were found to be significantly different and as such they were the only postoperative health outcomes considered for comparison in this study. Despite improvements in the treatment of incisional hernias, an average of $10 \%$ require surgical revision. ${ }^{12,16}$ In order to accurately represent the long-term costs incurred by incisional hernias, these revisions of laparotomies were also accounted for.

\section{Choice of perspective}

The CUA of small bite sutures was performed from the perspective of the NHS, since this perspective is recommended by NICE ${ }^{17}$ The alternative is a societal perspective, which would consider costs and benefits to the NHS, as well as accounting for indirect costs, such as the loss of productivity to society from work missed due to recovery from surgical complications. ${ }^{18}$ While a societal perspective allows for a more patient-centered analysis of costs, this introduces an element of subjectivity.

\section{Choice of comparator}

Currently, common practice is to perform closure of midline laparotomies with large bite sutures in order to protect against the effects of abdominal distension after surgery., ${ }^{4,12,19}$ Evidence suggests that a SL 4 times greater than the WL reduces 
the risk of incisional hernias. ${ }^{10}$ Therefore, in order to compare short bite sutures with large bite sutures, both techniques must have equal SL:WL ratios, meaning that their tension is similar and fewer large bite sutures are required than small bite sutures, as demonstrated in Figure 1. Consequently, the documented confounding effect that SL:WL ratios have on the incidence of incisional hernias postoperatively is controlled for. Therefore, the standard practice of large bites with a SL:WL ratio of 4 was used as the comparator against the proposed alternative method of short bites of an equal SL:WL ratio.

\section{Choice of horizon}

Surgeries produce long-term effects that are not immediately recognized. The complications that occur from surgery are negative externalities of production, leading to deadweight social loss, suggesting that the services of health care are not being allocated efficiently. To take into account all costs and benefits accurately, a specific time period was set in which subsequent costs were measured in order to account for these negative externalities.

Studies show that the majority of incisional hernias present within 3 years after the surgery, with at least $50 \%$ discovered within 2 years of surgery. ${ }^{2,5,8}$ Therefore, to take into account as many hernias that could be feasibly observed, the time horizon was set at 3 years. Only primary recurrences of incisional hernias were examined. Subsequent recurrences would be outside of the analytic horizon and would introduce multiple confounders, leading to an overestimation of costs.

\section{Literature review}

A literature review was conducted to identify important costs, complication rates, clinical effectiveness and probability measures relating to small and large bites. A search string was defined on the basis of the aims of the study using the population, intervention, comparator, outcome method (Table S1). Ovid, Medline and Cochrane databases were searched. The search string and corresponding number of results is outlined in Table $\mathrm{S} 2$.

Strict inclusion and exclusion criteria were applied. All included studies were screened to ensure that they were peer reviewed and their methods were assessed to ensure the validity of the results. Studies that were not published in English, or did not study adults and humans, were excluded. Included papers were screened by title and abstract to ensure that only studies that compared small and large bite sutures for midline laparotomies were included. Subsequent inclusions then underwent full-text review against the same criteria. Additional studies were then added through the process of snowball sampling by searching references from important studies. This process is outlined in the PRISMA diagram depicted in Figure S1. ${ }^{20}$

A total of 35 articles were identified that related to the suturing technique for midline laparotomies and incidence of incisional hernia or infection. Of these, only three articles were randomized controlled trials that directly compared small suture bites with large suture bites. ${ }^{5,7,9}$ Therefore, only these studies were deemed to be of high enough quality to provide data regarding incisional hernia incidence and SSI incidence with small and large bites. All three trials were conducted in different study populations and analyzed suture
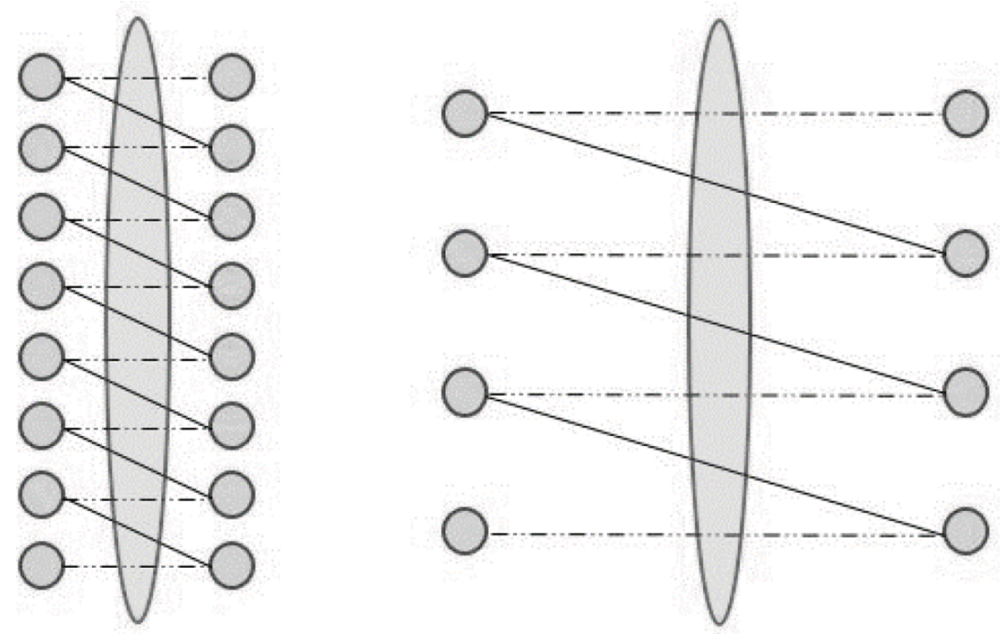

Figure I Diagram representing the difference between small bites and large bites with an equal SL:WL ratio.

Note: The definition/explanation of the term 'small bites and large bites with an equal SL:WL ratio' was taken from Millbourn D. ${ }^{4}$

Abbreviation: SL:WL, suture length to wound length. 
technique for differing operations within differing specialties, and so were not directly comparable. Consequently, postoperative complication data were drawn from the largest trial with the longest follow-up period. ${ }^{7}$ However, the results of the remaining two trials were compared and $95 \%$ confidence intervals (CIs) were extracted to provide a full range of parameter values for the sensitivity analysis.

In addition, one further study was an economic evaluation comparing the use of small and large bites in Sweden. ${ }^{8}$ Data from this study were extracted to provide indications of the cost implications of small bites, but figures were adjusted using NHS costs and data from NICE in order to be representative of UK costs. These studies are summarized in Table S3. Therefore, due to the limited published material pertaining to this area of study, the majority of data about the outcome probabilities and QALYs were based on non-UK European populations, eg, from Sweden and the Netherlands.

\section{Postoperative complications data}

Data on postoperative complications were synthesized from the literature review. Deerenberg et al's randomized controlled trial comparing small to large bite suture techniques identified that the use of small bite sutures is not associated with an increase in adverse events or postoperative pain. ${ }^{5}$ The study advocates the use of small bite sutures as the new standard for fascial closure of midline incisions. This conclusion is supported by Millbourn's analysis of midline laparotomy closure methods and their wound complications in Sweden. ${ }^{4}$ The latter recognizes a $35 \%$ relative reduction in rates of incisional hernias with the use of small bites compared to the standard large bite technique (11\% vs 17\%).

The majority of incisional hernias occur in the first year post-operation but can take up to 10 years to appear. ${ }^{21}$ Incidence rates reported in literature for hernias vary due to different definitions used. In this study, the European Hernia Society definition of an incisional hernia was used to ensure that the appropriate papers were analyzed. This defines an incisional hernia as "any abdominal wall defect, with or without a bulge, in the area of the scar perceptible or palpable by clinical examination or imaging". ${ }^{22}$ Incisional hernias are a burden both to the patient and society due to the pain that they can cause and the mobility problems associated with them. ${ }^{5,12}$ Furthermore, $36 \%$ require surgical repair due to the risks of incarceration and strangulation. ${ }^{4,16,23,24}$ Mesh repairs are the recommended option for most patients, but can be very expensive and have recurrence rates of up to $20 \%$. Thus, incisional hernias can have significant implications, both on patients' quality of life and costs to the NHS. ${ }^{12}$

SSIs are the second most common postoperative complication to affect midline laparotomies, ${ }^{12}$ leading to pain and longer stays in hospital. ${ }^{25}$ Furthermore, SSIs can double the risk of incisional hernias. ${ }^{7,26,27}$ The literature supports the use of small bites, which are shown to reduce the risk of SSI by $50 \% .^{7,26}$ All probabilities and incidence rates used in the calculations are collated in Table 1 . The data are adapted from studies performed by Deerenberg et al, ${ }^{5}$ Millbourn et al, ${ }^{7}$ Zhang et al, ${ }^{28}$ Muysoms et al, ${ }^{29}$ Flum et al $^{16}$ and Murray et al. ${ }^{27}$

\section{Quality-adjusted life year data}

The availability of QALY data associated specifically with cosmesis and pain after small bite and large bite closure in midline laparotomies was limited. However, previous studies have indicated that quality of life scores for small bite and large bite patients do not differ significantly in any domain except for mobility, in which small bite patients experience a better quality of life. ${ }^{5}$ QALY data concerning incisional hernias and SSIs were extracted from a CUA of incisional hernia repair methods by Fischer et al. ${ }^{30}$ Fischer et al conducted a survey and literature review of studies of patients with an average age of 50 years, and assumed an average life expectancy of 80 years. Since the average age of patients in the studies used to indicate effectiveness of small bites was

Table I Probabilities

\begin{tabular}{|c|c|c|c|}
\hline Parameter & $\begin{array}{l}\text { Minimum } \\
\text { probability }\end{array}$ & $\begin{array}{l}\text { Base case } \\
\text { probability }\end{array}$ & $\begin{array}{l}\text { Maximum } \\
\text { probability }\end{array}$ \\
\hline Rate of incisional hernia following small bite closure in SSI-free patients & NA & $0.047^{7}$ & NA \\
\hline Rate of hernia following large bite closure in SSI-free patients & NA & $0.172^{7}$ & NA \\
\hline Absolute percentage reduction in rate of hernia due to small bite closure in SSI-free patients & $0.027^{5}$ & $0.125^{7}$ & $0.405^{9}$ \\
\hline Rate of SSI in patients following small bite closure & NA & $0.051^{7}$ & NA \\
\hline Rate of SSI in patients following large bite closure & NA & $0.096^{7}$ & NA \\
\hline Absolute percentage reduction in rate of SSI due to small bite closure & $0.009^{9}$ & $0.045^{7}$ & $0.154^{9}$ \\
\hline Proportion of patients presenting with incisional hernias that require repair & $0.360^{16}$ & $0.360^{16}$ & $0.360^{16}$ \\
\hline Increased risk of developing an incisional hernia following SSI & $1.30^{27}$ & $1.93^{27}$ & $3.80^{27}$ \\
\hline Proportion of repairs requiring revision & $0.006^{34}$ & $0.100^{16}$ & $0.231^{16}$ \\
\hline
\end{tabular}

Abbreviations: NA, not applicable; SSI, surgical site infection. 
65 years, the QALY data from Fischer et al were adjusted appropriately to reflect this difference in age. QALYs were discounted by $3 \%$ annually. These values were combined with the rates of occurrence and recurrence of hernias and SSIs identified earlier.

\section{Cost data}

The cost of laparotomy is difficult to calculate with precision, as laparotomy is not an individual type of surgery and is indicated for multiple different surgical conditions. Overall, this cost is estimated at $£ 3,000^{31-33}$ based on multiple estimates. This figure is used throughout the analysis as a constant to allow the financial comparison of the two closure types. However, it should be noted that the study was comparing the difference in costs between the two techniques, and hence the actual cost of a laparotomy is a representative constant and would not influence the overall results of the study.

When estimating other costs, the most conservative figures found were used to ensure that cost savings were not overinflated. Small bite sutures increase the mean suturing time by 4.6 minutes per surgery. ${ }^{4}$ However, mean operating time has not been shown to differ significantly between the two groups. Following a conservative approach, costs of this potentially increased operating time were estimated at $£ 92$, using data from the NHS Institute for Innovation and Improvement. ${ }^{33}$ It should be noted that this cost primarily includes added staff time of less than 20 minutes in theatre per operating day. Staff may be on fixed salaries that would not necessarily rise in proportion to these extra minutes of work per day. Nevertheless, in the interests of producing a conservative cost comparison, this nuance was not taken into account.

This cost was evaluated against the standard costs of treating any potential postoperative complication that may occur after using large bite sutures for fascial closure, such as outpatient clinic appointments and surgical revisions. ${ }^{34}$
Therefore, a range of randomized controlled trials and systematic reviews were used to estimate these costs, an overview of which can be found in Table 2. When rounding, all figures were rounded in the direction that reduced costeffectiveness. It was assumed that patients who developed an incisional hernia that did not require treatment had no additional cost implications to the NHS, again in order to be as conservative as possible.

\section{Discounting factor}

Due to the time value, or the potential earning capacity, of money, money available at the present time is worth more than the same amount in the future. This "positive time preference" is due in part to the diminishing marginal utility of income. This study considered costs over a 3-year time period. Discounting costs over this time ensures that consideration is paid to the social opportunity costs of healthcare investments and ensures appropriate benchmarks for return on investments are met. ${ }^{35}$

NICE recommends discounting at an annual rate of $3.5 \%$ for costs incurred more than 1 year after an intervention. ${ }^{36}$ Since added costs incurred through fascial closure with small bites and the costs of treating a SSI occur within the first year, these were not discounted. Studies show that the majority of incisional hernias occur within 3 years of a midline laparotomy. ${ }^{26,37}$ Of these, an estimated $40 \%$ occur within 1 year, another $40 \%$ within the next year and $20 \%$ within the 3rd year after a laparotomy. ${ }^{38}$ It was assumed that the hernias requiring repair and those that recur would also follow this trend, and so the costs of repairing hernias was discounted in line with this. This is detailed in Table 2. Since the majority of costs occurred within 1 year, the analysis was not sensitive to the effects of discounting.

QALY data were not discounted by the authors as Fischer et $\mathrm{al}^{30}$ had already appropriately discounted the data in their analysis.

Table 2 Costs and justifications

\begin{tabular}{|c|c|c|c|c|}
\hline \multicolumn{4}{|l|}{ Cost statistic } & \multirow[t]{2}{*}{ Justification } \\
\hline & Minimum & Base case & Maximum & \\
\hline $\begin{array}{l}\text { Cost of additional operating } \\
\text { theatre time allocated to small } \\
\text { bite sutures }\end{array}$ & $£ 604,8,33$ & $£ 92^{4,8,33}$ & $E \mid 24^{4,8,33}$ & $\begin{array}{l}\text { Mean increase in suturing time: } 4.6 \text { minutes per } \\
\text { laparotomy, cost of extra suturing time: } £ 20 \text { per minute }{ }^{4,8,33}\end{array}$ \\
\hline $\begin{array}{l}\text { Cost of treating surgical site } \\
\text { infections }\end{array}$ & $£ 409^{39}$ & $£ 409^{39}$ & $£ 409^{39}$ & NICE $2004^{39}$ \\
\hline Total cost of a hernia repair & $£ \mid, 484^{4,16,34,40,41}$ & $£ I, 790^{4,16,34,40,4 I}$ & $£ 2, \mid 38^{4,16,34,40,4 \mid}$ & $\begin{array}{l}\text { Cost of incisional hospital hernia repair }(£|, 3| 8) \text {, plus } \\
\text { cost of } 3.5 \text { outpatient clinic appointments }(£ 354),{ }^{4,40,41} \text { plus } \\
\text { discounted added cost of laparotomies requiring revision } \\
(£ \mid I 8)^{16,34}\end{array}$ \\
\hline Cost of laparotomy & NA & $£ 3,000^{31-33}$ & NA & King et al, ${ }^{31}$ Morris et al, ${ }^{32}$ UK NHS Innovation ${ }^{33}$ \\
\hline
\end{tabular}

Abbreviations: NA, not applicable; NICE, National Institute of Health and Clinical Excellence; UK NHS, United Kingdom National Health Service. 


\section{Sensitivity analysis}

The data in the analysis are supported by the literature; however, those factors that have the greatest impact on the ICER are the clinical effectiveness probabilities for small bites, with respect to the decreased proportion of SSIs and incisional hernias seen in comparison to large bites.

A two-way sensitivity analysis was conducted to assess the combined impact of a variation in the clinical effectiveness of small bites with respect to SSIs and incisional hernias simultaneously. As the two variables are linked and have both synergistic and independent effects on the ICER, such an approach provides a more comprehensive and accurate picture. Additionally, although these important statistics are the result of multiple trials in Europe, they have yet to be verified by trials in the UK, and so assessment of the costutility of a range of combinations of the parameters helps to clarify the impact of the uncertainty around these estimates.

Threshold analysis was also conducted by way of a oneway sensitivity analysis of the impact of a change in the added cost of using small bites on their cost-utility.

\section{Results}

\section{Cost-utility analysis}

The probabilities, associated costs and QALY estimates identified from the literature review were synthesized to form a decision tree (Figure 2), which was used to calculate an ICER using large bites as the best available alternative treatment. The NICE threshold for clinical interventions is set between $£ 20,000$ and $£ 30,000$ per QALY. ${ }^{42}$

When evaluating the added costs of using short bites sutures against the quality of life improvements obtained by reducing SSIs and incisional hernias, short bites provide an ICER of $£ 6,390$ per QALY. However, this figure excludes the cost savings produced by reducing complication rates. Therefore, the true mean ICER for comparing short bites to large bites is -£482.61 per QALY. The calculation used is displayed in Figure 3.

Thus, the proposed intervention is not only cost-effective but also cost saving. There are also significant quality of life benefits compared to standard practice. This is displayed in Figure 4.

\section{Sensitivity analysis}

It should be noted that the ICER is only affected by a change in absolute SSI or hernia rates between small bites and large bites, and the effects of this change are constant regardless of the underlying rates (eg, reducing SSI rates from $10 \%$ to $8 \%$ provides the same ICER as a reduction from $12 \%$ to $10 \%$ ).
Figure 5 shows that small bites are a cost-effective intervention (when using the conservative NICE threshold of $£ 20,000 / \mathrm{QALY}$ ) when they reduce either the absolute rate of SSIs by more than $15 \%$ or the absolute rate of hernias by more than $3.4 \%$, or reduce both rates by a smaller amount in certain combinations.

The base case falls well within this threshold, and 95\% CIs for the parameters also fall within the plausible region, suggesting that all other factors held equal; small bites are likely to be cost-effective across the entire range of clinical thresholds.

The one-way sensitivity analysis of the impact of a change in the added cost of using small bites on their cost-utility is shown in Figure 6.

The $95 \%$ CIs for the increased suturing time for small bites ranged from 2 to 6 minutes, providing a cost range of $£ 40-£ 144$ per patient. This range is well below the $£ 20,000$ / QALY threshold, although the intervention is not cost neutral toward the upper bound. This indicates that, as long as the added cost of using small bites is less than $£ 386$ per patient, the intervention is cost-effective; however, the added cost must be below $£ 98$ per patient for small bites to also be cost-neutral.

\section{Discussion}

This CUA has demonstrated that the use of small bite sutures is both financially and clinically beneficial when compared to large bite sutures in midline laparotomies. The study estimates a cost saving of $£ 483$ per QALY gained, a value well below the threshold set by NICE for such analyses. Therefore, the UK NHS would benefit from a change to current practice by moving from large suture bites placed $10 \mathrm{~mm}$ from the wound edge to small bites placed 3-6 $\mathrm{mm}$ from the wound edge, given all other factors are held equal. The resultant decrease in SSIs and hernias far outweighs any additional suturing time.

The sensitivity analysis demonstrated that small bites are also cost-effective in the worst-case scenarios. The study conclusions of cost-effectiveness are not changed by variations of key parameters within their plausible range. Multiple studies have shown that small bites are unlikely to cost more than $£ 144$ per patient; ${ }^{4,8}$ however, the intervention would still be cost-effective even if it costs 3 times this amount. This is despite the fact that, in the short run, costs are unlikely to rise due to additional suturing time. The major expense would be additional staffing costs, but surgeons and other staff are likely to be on fixed salaries as 


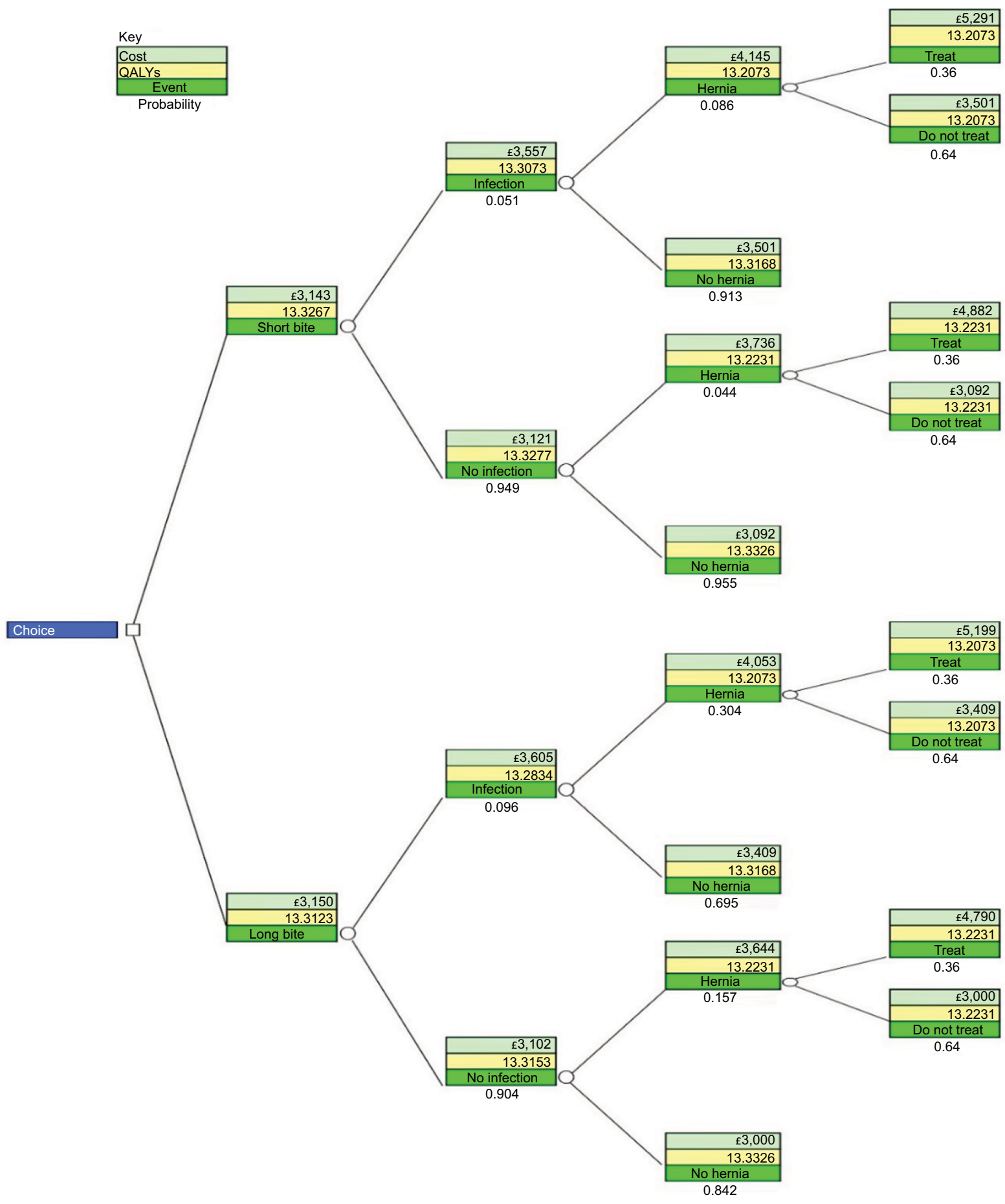

Figure 2 Decision tree.

opposed to hourly wages. The 4.6 minutes added suturing time is also only a small proportion of the overall length of laparotomy. ${ }^{4}$

Additionally, the sensitivity analysis also shows that, at an additional cost of $£ 92$ per patient, small bites are costeffective provided that they decrease absolute SSI rates by more than $15 \%$, or reduce absolute incisional hernia rates by more than $3.4 \%$. Studies in this area have shown that both incisional hernia and SSI rates are reduced by small bites ${ }^{4,5}$ resulting in a synergistic effect. For example, in order to reach the cost-effectiveness threshold of $£ 20,000$ / QALY, the absolute SSI rate needs to be reduced by only $7 \%$ if incisional hernia rates are also reduced by $2 \%$. It is worth noting, however, that in centers where incisional 


$$
\begin{gathered}
\text { ICER }=\frac{\text { Cost }_{\text {small bites }}-\text { Cost }_{\text {large bites }}}{\text { Effects }_{\text {small bites }}-\text { Effects }_{\text {large bites }}}=\frac{3.143-3,150}{13.3267-13.3123}=\frac{-6.95}{0.0144} \\
=-£ 482.61 \text { per QALY }
\end{gathered}
$$

(Subject to rounding)

Figure 3 ICER calculation.

Abbreviations: ICER, incremental cost-effectiveness ratio; QALY, quality-adjusted life year.

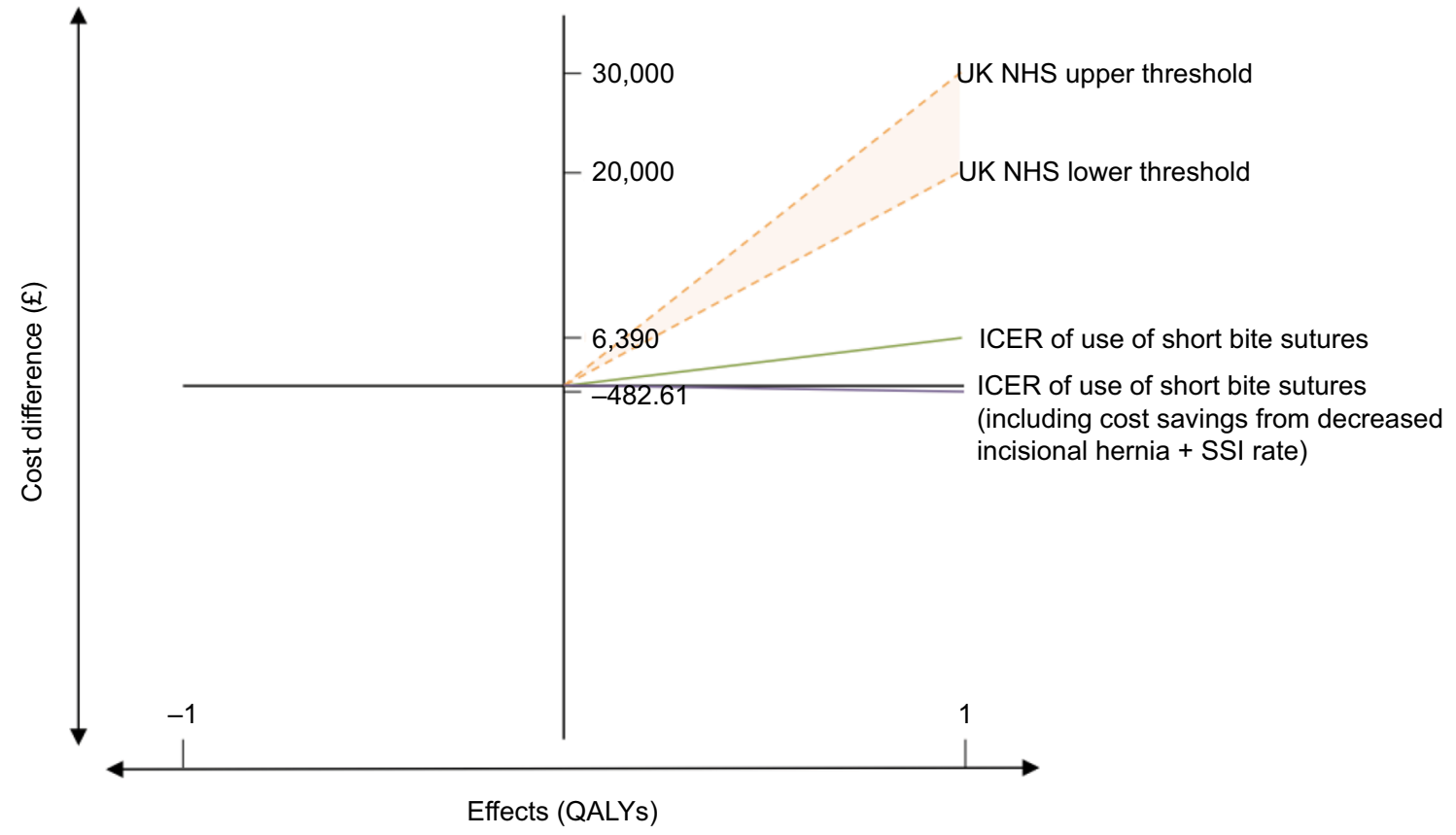

Figure 4 Graph displaying ICER of short bite sutures alongside NHS thresholds.

Abbreviations: ICER, incremental cost-effectiveness ratio; QALY, quality-adjusted life year; UK NHS, United Kingdom National Health Service; SSI, surgical site infection.

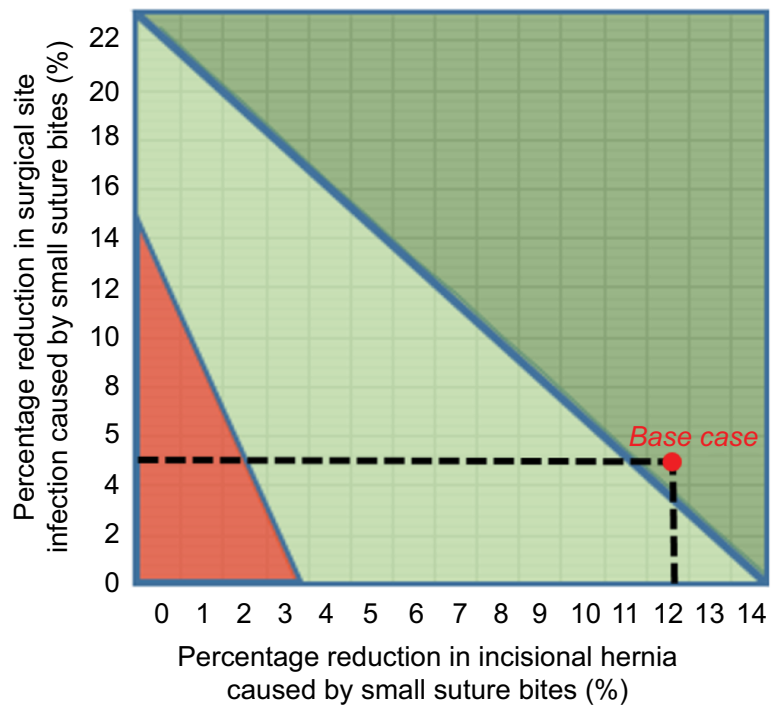

Key:
ICER $<£ 0 / Q A L Y$ (intervention is cost neutral)
$£ 20,000<$ ICER $£ 0 / Q A L Y$ (intervention is
cost-effective)
ICER $<£ 20,000 / Q A L Y$ (intervention is not
cost-effective)

Figure 5 Graph displaying boundaries for percentage change in both outcomes with respect to ICER limits.

Abbreviations: ICER, incremental cost-effectiveness ratio; QALY, quality-adjusted life year. 


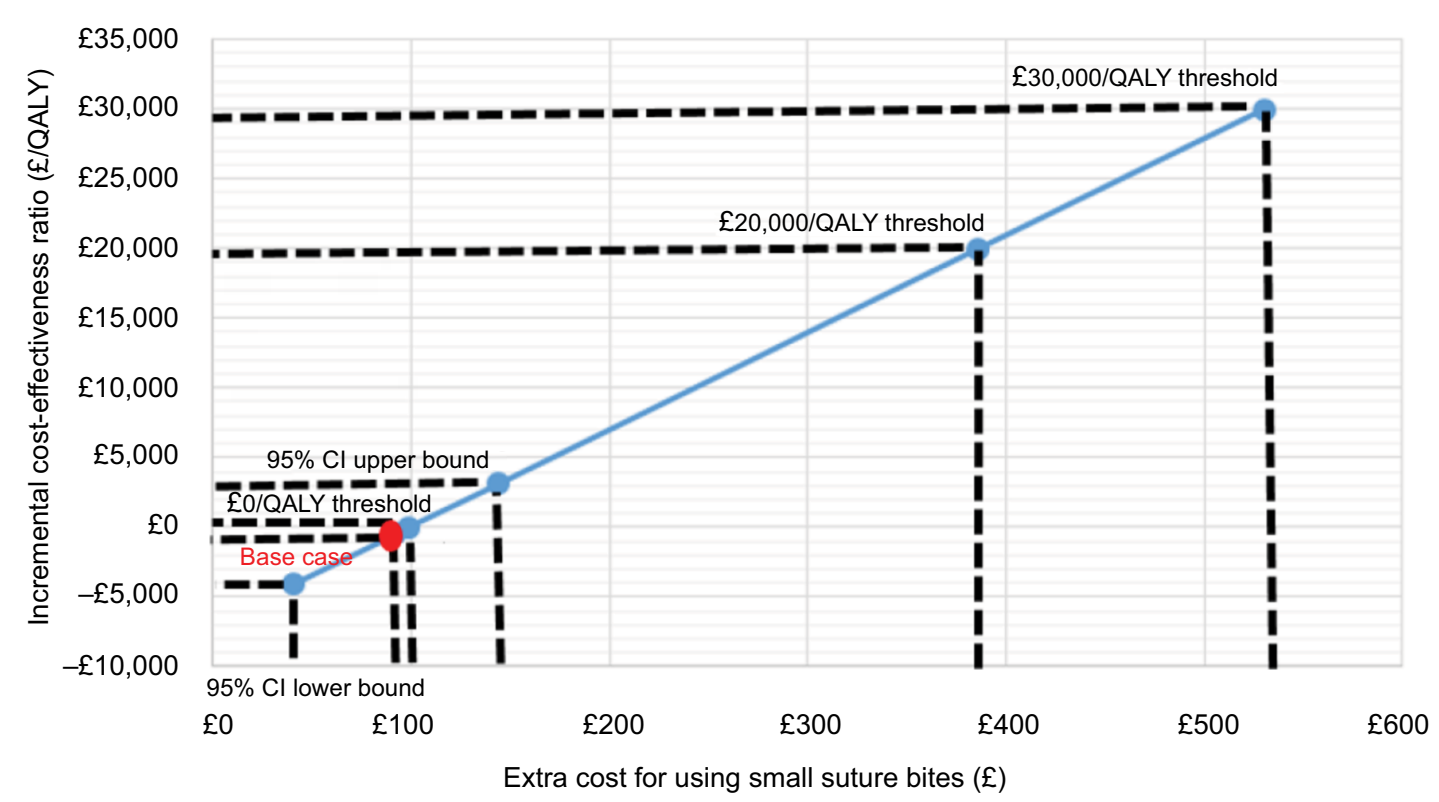

Figure 6 One-way sensitivity analysis.

Abbreviations: $\mathrm{Cl}$, confidence interval; QALY, quality-adjusted life year.

hernia and SSI rates are both close to zero, small bites are far less likely to be cost-effective.

The results of this study parallel Millbourn et al's conclusion that utilizing small bite sutures is associated with a cost reduction. ${ }^{8}$ However, Millbourn et al's study was conducted from a Swedish perspective and only used data from one randomized controlled trial conducted in 2012. By contrast, this study applied the data to the UK perspective, as well as including data from other trials, such as that conducted by Deerenberg et al..$^{5}$ Other research is minimal, especially in the UK where there has been no related research, and so comparison to additional literature is limited. Additionally, this study has also quantified the threshold levels for cost-utility in terms of SSI reduction rates, incisional hernia reduction rates and additional operating costs, which is yet to be seen in other studies.

With 40,000 emergency midline laparotomies performed each year in the UK, ${ }^{3}$ the adoption of small suture bites could save the UK NHS $£ 278,000$ per year, while simultaneously generating 576 QALYS per year. Importantly, this figure does not consider elective laparotomies, which would also contribute significantly to these benefits. However, the aim of this study was to accurately quantify cost-utility, but not to accurately quantify the total annual savings to the NHS, as data were not available on the exact number of operations that would be suitable for small suture bites. Hence, further research is needed to validate these figures.

Additionally, various limitations were encountered when investigating the cost-utility of small bites in midline laparotomies. First and foremost, the analysis relied on evidence from outside the UK. Further research on the effectiveness of small bites sutures in the UK is required to ensure that the quantification of benefits is wholly applicable to the UK population. In order to mitigate this, the sensitivity analysis quantified threshold values for complication rates to ensure that when such research is conducted, this analysis can be reinterpreted in light of new data on complication rates.

Second, laparotomies encompass several different types of surgery, all with varying postoperative complication types and rates. The range of indications for laparotomy also reflects the range of the pre-existing quality of life of patients undergoing this type of surgery. The relative effects of postoperative complications may therefore vary depending on the pre-existing quality of life of the individual patients. While this analysis used average data for QALYs, further research is required in order to quantify cost-utility accurately for each type of laparotomy.

Third, this CUA did not take into account the full social costs and benefits of SSIs or incisional hernias, such as 
working days lost or costs of care within the community. Additionally, only the two most important factors of SSIs and incisional hernias were taken into account. Other factors such as wound dehiscence, bowel obstruction, cosmesis and pain were not considered since they did not vary significantly between small bites and large bites. ${ }^{5}$ Quantifying their impact on cost-utility would add a level of complexity to the analysis, which was outside the scope of this study.

Finally, there would be costs associated with the implementation of small suture bites since this involves a change of practice. Healthcare providers would need to be retrained to adopt this technique and surgeons would need to be educated to keep SL:WL ratio the same. However, a culture of resistance amongst experienced and trained surgeons may hinder the adoption of the proposed technique. Furthermore, this training would possibly take up more time, adding to the existing time pressures placed on surgeons. Further work is required to quantify these costs, although a formal health technology assessment could utilize the results of this CUA when considering whether to encourage surgeons to make small bites sutures as the standard practice.

\section{Impact and scope}

This study suggests that using small bite sutures for midline laparotomies in the UK is both cost saving and cost-effective. The study therefore advocates a change in practice in UK guidelines for the closure of midline incisions in midline laparotomies. Since midline laparotomies are a common procedure, standardized suturing techniques have the ability to generate large savings for the NHS. While the clinical effectiveness of this technique has already been proven, it is important that cost-effectiveness is also considered. There is also further scope to investigate the application of the proposed technique to other types of surgery.

\section{Conclusion}

This study demonstrates that the small bite suture technique is a cost-effective alternative to the current practice of using large bite sutures in the closure of midline laparotomies. This technique has the potential to reduce the financial burden within the NHS and lead to better outcomes for patients. Further research in this area will enable the results of this work to be validated for specific types of surgery.

\section{Acknowledgment}

The authors wish to thank Robert Morton, Meelad Sayma, and Manraj Singh Sura for their assistance in structuring this work.

\section{Disclosure}

The authors report no conflicts of interest in this work.

\section{References}

1. Patnalk VVG, Singla RK, Bansal VK. Surgical incisions - their anatomical basis: part IV-abdomen. J Anat Soc India. 2001;50(2):170-178.

2. Burger JW, van't Riet M, Jeekel J. Abdominal incisions: techniques and postoperative complications. Scand J Surg. 2002;91(4):315-321.

3. Barrow E, Anderson ID, Varley S, et al. Current UK practice in emergency laparotomy. Ann R Coll Surg Engl. 2013;95(8):599-603.

4. Millbourn D. Closure of Midline Abdominal Incision with Small Stitches: Studies on Wound Complications and Health Economy [doctoral thesis]. Umeå: Umeå University; 2012.

5. Deerenberg EB, Harlaar JJ, Steyerberg EW, et al. Small bites versus large bites for closure of abdominal midline incisions (STITCH): a double-blind, multicentre, randomised controlled trial. Lancet. 2015;386(10000):1254-1260.

6. Gejoe G, Yadev I, Rahul M. Emergency laparotomies at a tertiary care center - a hospital-based cross-sectional study. Indian J Surg. 2017;79(3):206-211.

7. Millbourn D, CengizY, Israelsson LA. Risk factors for wound complications in midline abdominal incisions related to the size of stitches. Hernia. 2011;15(3):261-266.

8. Millbourn D, Wimo A, Israelsson LA. Cost analysis of the use of small stitches when closing midline abdominal incisions. Hernia. 2013;18(6):775-780.

9. Millbourn D, CengizY, Israelsson LA. Effect of stitch length on wound complications after closure of midline incisions: a randomized controlled trial. Arch Surg. 2009;144(11):1056-1059.

10. Israelsson LA, Jonsson T. Suture length to wound length ratio and healing of midline laparotomy incisions. Br J Surg. 1993;80(10):1284-1286.

11. Israelsson LA, Jonsson T. Overweight and healing of midline incisions: the importance of suture technique. Eur J Surg. 1997;163(3): $175-180$.

12. Sanders DL, Kingsnorth AN. The modern management of incisional hernias. BMJ. 2012;344:e2843.

13. Sanders RJ, DiClementi D, Ireland K. Principles of abdominal wound closure. I. Animal studies. Arch Surg. 1977;112(10):1184-1187.

14. Harlaar JJ, van Ramshorst GH, Nieuwenhuizen J, et al. Small stitches with small suture distances increase laparotomy closure strength. $\mathrm{Am}$ J Surg. 2009;198(3):392-395.

15. Remme M, Martinez-Alvarez M, Vassall A. Cost-effectiveness thresholds in global health: taking a multisectoral perspective. Value Health. 2017;20(4):699-704.

16. Flum DR, Horvath K, Koepsell T. Have outcomes of incisional hernia repair improved with time? A population-based analysis. Ann Surg. 2003;237(1):129-135.

17. Monahan M, Barton P, Taylor CJ, et al. MICE or NICE? An economic evaluation of clinical decision rules in the diagnosis of heart failure in primary care. Int J Cardiol. 2017;241:255-261.

18. Byford S, Raftery J. Perspectives in economic evaluation. BMJ. 1998;316(7143):1529-1530.

19. Jenkins TP. The burst abdominal wound: a mechanical approach. $\mathrm{Br} J$ Surg. 1976;63(11):873-876.

20. Moher D, Liberati A, Tetzlaff J, Altman DG; The PRISMA Group. Preferred reporting items for systematic reviews and meta-analyses: the PRISMA statement. BMJ. 2009;339:b2535.

21. Slater NJ, Bleichrodt RP, van Goor H. Wound dehiscence and incisional hernia. Surgery (Oxford). 2012;30(6):282-289.

22. Muysoms FE, Miserez M, Berrevoet F, et al. Classification of primary and incisional abdominal wall hernias. Hernia. 2009;13(4):407-414.

23. Mudge M, Hughes LE. Incisional hernia: a 10 year prospective study of incidence and attitudes. Br J Surg. 1985;72(1):70-71.

24. Israelsson LA, Wimo A. Cost minimisation analysis of change in closure technique of midline incisions. Eur J Surg. 2000;166(8):642-646. 
25. Coello R, Charlett A, Wilson J, Ward V, Pearson A, Borriello P. Adverse impact of surgical site infections in English hospitals. J Hosp Infect. 2005;60(2):93-103.

26. Bucknall TE, Cox PJ, Ellis H. Burst abdomen and incisional hernia: a prospective study of 1129 major laparotomies. Br Med J (Clin Res Ed). 1982;284(6320):931-933.

27. Murray BW, Cipher DJ, Pham T, Anthony T. The impact of surgical site infection on the development of incisional hernia and small bowel obstruction in colorectal surgery. Am J Surg. 2011;202(5):558-560.

28. Zhang MX, Sun YH, Xu Z, Zhou P, Wang HX, Wu YY. Wound edge protector for prevention of surgical site infection in laparotomy: an updated systematic review and meta-analysis. ANZ J Surg. 2015;85(5):308-314.

29. Muysoms FE, Antoniou SA, Bury K, et al; European Hernia Society. European Hernia Society guidelines on the closure of abdominal wall incisions. Hernia. 2015;19(1):1-24.

30. Fischer JP, Basta MN, Wink JD, Krishnan NM, Kovach SJ. Cost-utility analysis of the use of prophylactic mesh augmentation compared with primary fascial suture repair in patients at high risk for incisional hernia Surgery. 2015;158(3):700-711.

31. King PM, Blazeby JM, Ewings P, et al. Randomized clinical trial comparing laparoscopic and open surgery for colorectal cancer within an enhanced recovery programme. Br J Surg. 2006;93(3):300-308.

32. Morris S, Gurusamy KS, Sheringham J, Davidson BR. Cost-effectiveness of diagnostic laparoscopy for assessing resectability in pancreatic and periampullary cancer. BMC Gastroenterol. 2015;15:44.

33. NHS Innovation and Improvement. Improving quality and efficiency in the operating theatre: a lifeline for financial leaders [Internet]. Coventry: NHS Innovation and Improvement; 2009. Available from http://harmfreecare.org/wp-content/files_mf/Improving-quality-andefficiency-in-the-operating-theatre.pdf. Accessed August 27, 2017.

34. Andersen LPH, Klein M, Gögenur I, Rosenberg J. Long-term recurrence and complication rates after incisional hernia repair with the open onlay technique. BMC Surg. 2009;9:6.
35. Claxton K, Sculpher M, Culyer A, et al. Discounting and costeffectiveness in NICE - stepping back to sort out a confusion. Health Econ. 2005;15(1):1-4.

36. National Institute for Health and Care Excellence. Guide to the methods of technology appraisal [homepage on the Internet]. London: NICE; 2013. Available from: https://www.nice.org.uk/process/pmg9/chapter/ further-information. Accessed August 27, 2017.

37. Lamont PM, Ellis H. Incisional hernia in re-opened abdominal incisions: an overlooked risk factor. Br J Surg. 1988;75(4):374-376

38. Brandl A, Laimer E, Perathoner A, Zitt M, Pratschke J, Kafka-Ritsch $\mathrm{R}$. Incisional hernia rate after open abdomen treatment with negative pressure and delayed primary fascia closure. Hernia. 2014;18(1): $105-111$.

39. National Institute for Health and Care Excellence. Surgical site infections: prevention and treatment. London: NICE; 2008 [updated February 2017]. Available from: https://www.nice.org.uk/guidance $/ \operatorname{cg} 74$ Accessed August 27, 2017.

40. Gheorghe A, Roberts TE, Pinkney TD, Bartlett DC, Morton D, Calvert M; West Midlands Research Collaborative; ROSSINI Trial Investigators. The cost-effectiveness of wound-edge protection devices compared to standard care in reducing surgical site infection after laparotomy: an economic evaluation alongside the ROSSINI trial. PLoS One. 2014;9(4):e95595.

41. Mazari FA, Thomas K, Yeung MM, Shiwani MH. Cost effectiveness of laparoscopic ventral \& incisional hernia repair. Poster presented at: the Society of American Gastrointestinal and Endoscopic Surgeons Meeting; April 17-20; 2013; Baltimore, MD, USA. Available from: https:/www.sages.org/meetings/annual-meeting/abstracts-archive/ cost-effectiveness-of-laparoscopic-ventral-incisional-hernia-repair/. Accessed August 27, 2017.

42. Waxman K. Building the business case for quality. In: Waxman K, editor. Financial and Business Management for the Doctor of Nursing Practice. New York: Springer; 2013:185. 


\section{Supplementary materials}

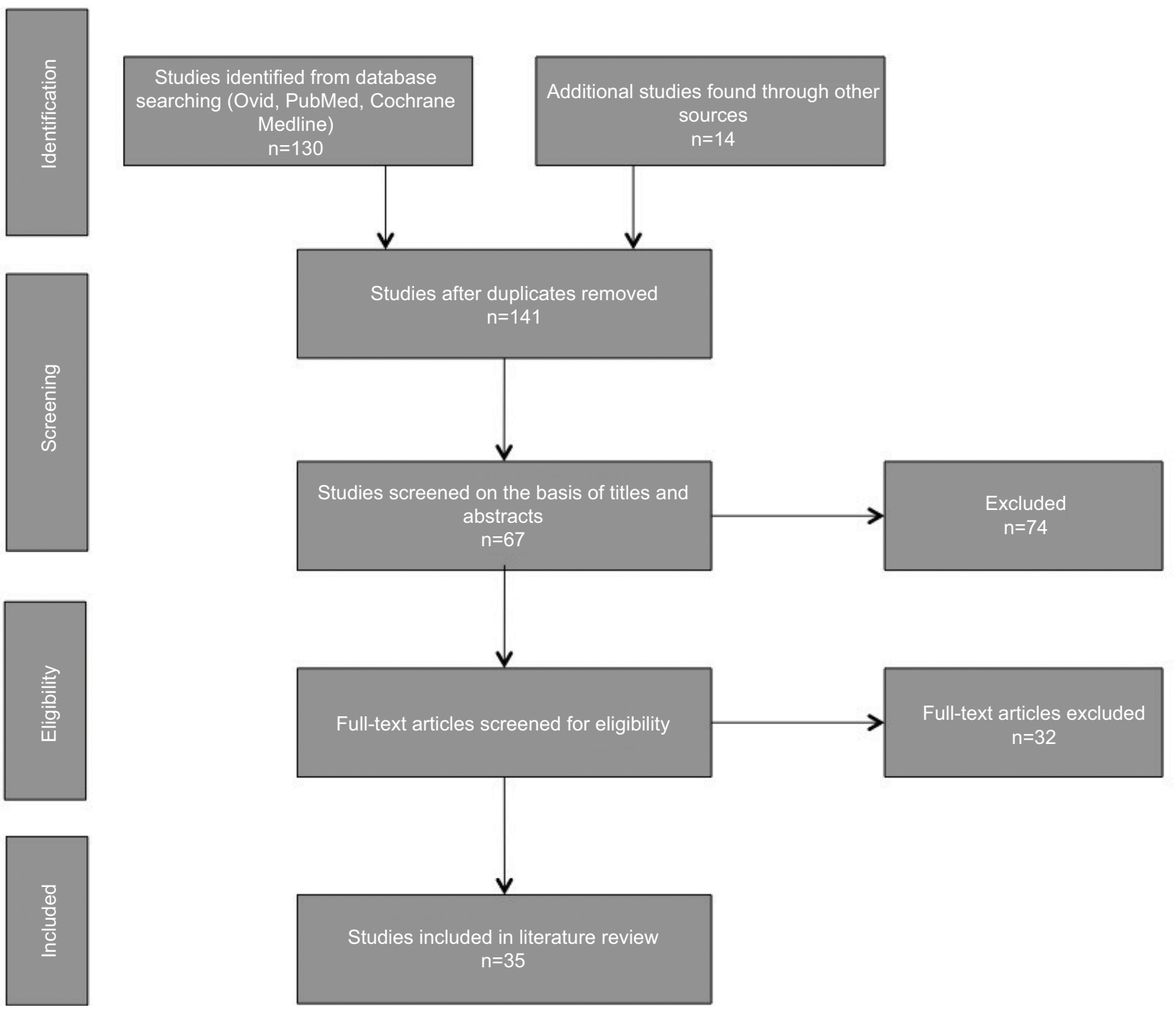

Figure SI PRISMA reporting diagram for literature review.

Note: Figure adapted from Moher D, Liberati A, Tetzlaff J, Altman DG; The PRISMA Group. Preferred reporting items for systematic reviews and meta-analyses: the PRISMA statement. BMJ. 2009;339:b2535.'

Abbreviation: PRISMA, preferred reporting items for systematic reviews and meta-analyses.

Table SI Search terms

\begin{tabular}{ll}
\hline Parameter & Description \\
\hline Population & Adult patients undergoing elective midline laparotomies \\
Intervention & Small bite sutures placed 3-6 mm from the wound edge with a SL:WL ratio of 4:I \\
Comparator & Large bite sutures placed $10 \mathrm{~mm}$ from the wound edge with a SL:WL ratio of 4:I \\
Outcome & Cost-utility (associated costs and QALYs, including SSI and incisional hernia rates) \\
\hline
\end{tabular}

Abbreviations: SL:WL, suture length to wound length; QALYs, quality-adjusted life years; SSI, surgical site infection.

Table S2 Search string

\begin{tabular}{ll}
\hline Search term & Number of results \\
\hline Abdominal incision OR laparotomy OR midline incision & 46,228 \\
(Suture OR sutures OR stitch) AND (large OR small OR long OR short) & 14,633 \\
Incisional hernia OR pain OR infection OR cosmesis OR death OR dehiscence OR rupture & $1,933,201$ \\
Combine search terms with “AND" function & 228 \\
Apply "English language," "human," and "all adult (I9 plus years)" filters & 130 \\
\hline
\end{tabular}


Table S3 Key studies from the literature review

\begin{tabular}{|c|c|c|c|}
\hline Reference & Title & Year published & Summary \\
\hline Deerenberg et $\mathrm{al}^{2}$ & $\begin{array}{l}\text { Small bites versus large } \\
\text { bites for closure of } \\
\text { abdominal midline incisions } \\
\text { (STITCH): a double-blind, } \\
\text { multicentre, randomised } \\
\text { controlled trial. }\end{array}$ & 2015 & $\begin{array}{l}\text { This prospective, multicenter, double-blind, randomized controlled } \\
\text { trial at } 10 \text { hospitals in the Netherlands compared small suture bites } \\
\text { to large bites to observe the occurrence of incisional hernias. Five } \\
\text { hundred and sixty patients were included in the study randomized } \\
\text { to either group. Data for the rate of complications in both groups } \\
\text { were extracted from this study up to its limit time of I year. }\end{array}$ \\
\hline Millbourn et $\mathrm{al}^{3}$ & $\begin{array}{l}\text { Risk factors for wound } \\
\text { complications in midline } \\
\text { abdominal incisions related } \\
\text { to the size of stitches. }\end{array}$ & 2011 & $\begin{array}{l}\text { This single-center, double-blind, randomized controlled trial } \\
\text { investigated the effects of using small bites compared to large bites } \\
\text { on the rate of wound complications. Three hundred and twenty-one } \\
\text { patients were randomized to closure with small stitches and } 370 \\
\text { with large bites. Data for the rate of complications in both groups } \\
\text { were extracted from this study. }\end{array}$ \\
\hline Millbourn et $\mathrm{al}^{4}$ & $\begin{array}{l}\text { Cost analysis of the use of } \\
\text { small stitches when closing } \\
\text { midline abdominal incisions. }\end{array}$ & 2013 & $\begin{array}{l}\text { This article looked at the potential cost savings generated by } \\
\text { using small stitches when closing midline abdominal incisions. A } \\
\text { prospective, single-center, randomized controlled trial conducted } \\
\text { over } 5 \text { years compared closure of midline incisions using small bites } \\
\text { with the use of large bites. Data for the rates of complications and } \\
\text { costs were extracted from this study. }\end{array}$ \\
\hline Fischer et $\mathrm{al}^{5}$ & $\begin{array}{l}\text { Cost-utility analysis of the } \\
\text { use of prophylactic mesh } \\
\text { augmentation compared } \\
\text { with primary fascial suture } \\
\text { repair in patients at high } \\
\text { risk for incisional hernia. }\end{array}$ & 2015 & $\begin{array}{l}\text { This analysis aimed to determine the cost-utility of using } \\
\text { prophylactic mesh to augment fascial incisions. Researchers used } \\
\text { a decision tree model to evaluate the cost-utility of using PMA } \\
\text { relative to primary suture closure after elective laparotomy. They } \\
\text { conducted a systematic review of the literature on PMA. QALY } \\
\text { data were extracted from this study. }\end{array}$ \\
\hline
\end{tabular}

Abbreviations: CUA, cost-utility analysis; PMA, prophylactic mesh augmentation; QALY, quality-adjusted life year.

\section{References}

1. Moher D, Liberati A, Tetzlaff J, Altman DG; The PRISMA Group. Preferred reporting items for systematic reviews and meta-analyses: the PRISMA statement. BMJ. 2009;339:b2535.

2. Deerenberg EB, Harlaar JJ, Steyerberg EW, et al. Small bites versus large bites for closure of abdominal midline incisions (STITCH): a double-blind, multicentre, randomised controlled trial. Lancet. 2015;386(10000):1254-1260.

3. Millbourn D, Cengiz Y, Israelsson LA. Risk factors for wound complications in midline abdominal incisions related to the size of stitches. Hernia. 2011;15(3):261-266.
4. Millbourn D, Wimo A, Israelsson LA. Cost analysis of the use of small stitches when closing midline abdominal incisions. Hernia. 2013;18(6):775-780.

5. Fischer JP, Basta MN, Wink JD, Krishnan NM, Kovach SJ. Cost-utility analysis of the use of prophylactic mesh augmentation compared with primary fascial suture refpair in patients at high risk for incisional hernia. Surgery. 2015;158(3):700-711.
ClinicoEconomics and Outcomes Research

\section{Publish your work in this journal}

ClinicoEconomics and Outcomes Research is an international, peerreviewed open-access journal focusing on health technology assessment, pharmacoeconomics and outcomes research in the areas of diagnosis, medical devices, and clinical, surgical and pharmacologica intervention. The economic impact of health policy and health systems

\section{Dovepress}

organization also constitute important areas of coverage. The manuscript management system is completely online and includes a very quick and fair peer-review system, which is all easy to use. Visit http://www.dovepress.com/testimonials.php to read real quotes from published authors. 